\title{
Molecular Descriptors and Topological Analysis of Cyclooctane Derivatives
}

\author{
Jia-bao Liu ${ }^{1}$ and Ting Zhang ${ }^{2}$ \\ ${ }^{1}$ Anhui Jianzhu University South Campus \\ ${ }^{2}$ Anhui Jianzhu University
}

December 15, 2021

\begin{abstract}
Cyclooctane is mainly used in the synthesis of cyclooctanone, cyclooctanol, caprolactam and octanoic acid. At the same time, it can also be used as an intermediate in organic synthesis and a chemical reagent. By discussing the resistance distance between any two points of cyclooctane derivative $\operatorname{Tn}(\mathrm{C} 8)$, some invariants about resistance distance are obtained, such as Kirchhoff index, multiplicative degree-Kirchhoff index, and additive degree-Kirchhoff index. Topological index can help scholars better understand some physical and chemical properties of compounds, and we obtain the closed expressions of valency-based topological indices for $\operatorname{Tn}(\mathrm{C} 8)$, such as $\mathrm{ABC}$ index, GA index, etc.
\end{abstract}

\section{Hosted file}

Molecular Descriptors and Topological Analysis \selectlanguage\{ngerman\} of Cyclooctane Derivatives.pdf available at https://authorea.com/users/336629/articles/549509-molecular-descriptors-andtopological-analysis-of-cyclooctane-derivatives 